Revue d'histoire de l'Amérique française

REYUE D.HISTOIRE DE L'AMÉRIQUE FRANÇAISE

\title{
GAUVREAU Michael et Ollivier HUBERT, dir., The Churches and Social Order in Nineteenth-and Twentieth-Century Canada (Montréal, McGill-Queen's University Press, coll. « McGill-Queen's Studies in the History of Religion ", $n^{0} 45$, 2006), xii-316 p.
}

\section{Mélanie Lanouette}

Volume 61, numéro 3-4, hiver-printemps 2008

Dossier Québec

URI : https://id.erudit.org/iderudit/019148ar

DOI : https://doi.org/10.7202/019148ar

Aller au sommaire du numéro

Éditeur(s)

Institut d'histoire de l'Amérique française

ISSN

0035-2357 (imprimé)

1492-1383 (numérique)

Découvrir la revue

Citer ce compte rendu

Lanouette, M. (2008). Compte rendu de [GAUVREAU Michael et Ollivier HUBERT, dir., The Churches and Social Order in Nineteenth- and

Twentieth-Century Canada (Montréal, McGill-Queen's University Press, coll. " McGill-Queen's Studies in the History of Religion ", $\left.n^{0} 45,2006\right)$, xii-316 p.] Revue d'histoire de l'Amérique française, 61(3-4), 575-577.

https://doi.org/10.7202/019148ar d'utilisation que vous pouvez consulter en ligne. 
l'«exemple» de phénomènes plus amples ne doit pas être pris au pied de la lettre.

À cela s'ajoutent de réels problèmes d'écriture. La structure du texte est parfois si déficiente, et ses principaux fils conducteurs si peu mis en valeur, qu'il faut un effort soutenu pour en reconstituer la pleine cohérence. Un travail d'édition plus appuyé aurait sans doute atténué cet inconvénient, en plus d'éliminer les ambiguiités syntaxiques qui brouillent régulièrement l'écriture. Malgré ses faiblesses, l'ouvrage de Gaumer et Desrosiers, qui remplit également une fonction commémorative, n'en offrira pas moins un outil de référence utile aux chercheurs spécifiquement intéressés aux questions de santé publique ou à l'évolution administrative des unités de la Faculté de médecine de l’Université de Montréal.

JULIEN PRUD'HOMME Centre interuniversitaire de recherche sur la science et la technologie

Université du Québec à Montréal

GAUVREAU Michael et Ollivier HUBERT, dir., The Churches and Social Order in Nineteenth- and Twentieth-Century Canada (Montréal, McGill-Queen's University Press, coll. «McGill-Queen's Studies in the History of Religion », $n^{\circ} 45,2006$ ), xii-316 p.

Cet ouvrage dirigé par Michael Gauvreau et Ollivier Hubert apporte une contribution majeure à l'historiographie religieuse canadienne. D’une part, il réunit des contributions de chercheurs du Canada anglais et du Québec, une rencontre rarement osée et qui doit, à mon sens, être renouvelée. D’autre part, il décloisonne les perspectives traditionnellement admises en proposant des comparaisons à plusieurs échelles: entre catholicisme et protestantisme surtout, mais aussi entre les dénominations protestantes, les provinces, la langue maternelle des locuteurs, les classes sociales, etc.

Dans leur introduction, dont on doit saluer l'ampleur et la profondeur, Gauvreau et Hubert ne se contentent pas de présenter les textes de l'ouvrage, ils les mettent plutôt en perspective pour en développer une intelligence globale. Le propos est dense, témoignant des avancées considérables de l'historiographie religieuse des dernières décennies. Traditionnellement étudiées du point de vue de la religion dite "officielle», les institutions religieuses ont longtemps été perçues comme gardiennes des traditions, et par définition réfractaires au changement et à la modernité. 
En plus d'opposer la religion de l'élite à la religion populaire, une lecture linéaire de l'histoire religieuse a en outre soutenu l'image de fidèles soumis à une autorité religieuse vouée, dès le $\mathrm{XIX}^{\mathrm{e}}$ siècle, à un déclin progressif, sous la poussée conjuguée de la Révolution industrielle et de l'émancipation graduelle des individus face au pouvoir religieux. Certes, il est vrai que l'industrialisation a provoqué des changements majeurs dans les rapports entre le social et le religieux mais, fondamentalement, l'ouvrage rappelle que les institutions religieuses demeurent un lieu de transactions multiples et complexes. Immergées dans leur environnement social, les Églises, tant protestantes que catholiques, sont en réalité des objets d'études privilégiés pour saisir l'ampleur et le sens des transformations sociales des $\mathrm{XIX}^{\mathrm{e}}$ et $\mathrm{xx}^{\mathrm{e}}$ siècles.

Les neuf textes de l'ouvrage déclinent, chacun à leur façon, l'idée que les Églises sont loin d'avoir été étrangères aux processus de modernisation de la société. Non seulement proposent-elles, voire édictent-elles des normes, mais aussi répondent-elles aux conjonctures socio-économiques, ajustant leur propre mode d'insertion en société, l'encadrement offert aux fidèles, le degré de latitude laissé aux laïques dans la gestion commune des rites et des œuvres. Loin d'être incompatible avec la modernité, le contrôle ecclésiastique en subit les contrecoups, en même temps qu'il participe à sa construction. À cet égard, les lectures qui sont faites ici des rapports entre modernité et Églises sont toutes en nuances. D’un côté, on montre comment les institutions religieuses participent à la diffusion des valeurs libérales (Christie) et se bureaucratisent graduellement, à l'image de l'État d'ailleurs (Hudon et Hubert; Routhier), et de l'autre, on rappelle à quel point l'adaptation des institutions à la modernité permet la consolidation et l'affirmation de l'autorité ecclésiale. Une illustration tout à fait exemplaire de ce phénomène est la confessionnalisation des institutions publiques au XIx ${ }^{\mathrm{e}}$ siècle, particulièrement marquée au Québec. Ce changement majeur découle d'une libéralisation de la vie religieuse, en même temps qu'il permet à l'Église catholique d'occuper en société une place beaucoup plus importante qu'auparavant. Dans les domaines éducatif et caritatif, par exemple, les œuvres et les institutions religieuses occupent alors l'avantscène de l'espace public (Caulier; Fecteau et Vaillancourt).

Les études proposées dans l'ouvrage ont en commun d'être soutenues par une démarche microhistorique qui reconnaît aux individus, clercs comme laïques, la capacité d'orienter les changements qui se déploient à l'époque dans les espaces religieux et social. En focalisant sur les pratiques et les situations concrètes, les différentes contributions des auteurs mon- 
trent bien à quel point l'autorité n'est pas l'apanage d'une seule élite cléricale. La notion de contrôle est ainsi revisitée pour reconnaître comment le rôle parfois hégémonique revendiqué par les Églises a aussi été soutenu par les collectivités, au nom d'une participation plus démocratique aux affaires religieuses. Des auteurs soulignent ainsi le rôle joué par les laïques dans la gestion des Églises (Draper), tandis que d'autres proposent une relecture des divisions sociales au sein des Églises protestantes notamment, de manière à montrer qu'elles sont bien plus complexes que ce que l'historiographie traditionnelle a pu laisser paraître (Lane). Les classes ouvrières, loin d'être laissées pour compte dans le monde industriel en développement, animent au contraire activement la vie et les institutions religieuses (Gauvreau). Cela devient par exemple perceptible quand on quitte la sphère strictement spirituelle pour constater à quel point les institutions religieuses sont aussi, et peut-être d'abord, des institutions sociales qui permettent de conforter les appartenances communautaires et les solidarités sociales (Perin).

L'image qui se dégage au sortir de la lecture de l'ouvrage est celle d'un champ historiographique à la fine pointe des interrogations contemporaines en histoire, de travaux innovants et de contributions inédites en histoire sociale et culturelle canadienne. Pour le lectorat québécois, le livre permet peut-être aussi de découvrir une historiographie canadienneanglaise riche et inspirante. En fait, il faut retenir de cet ouvrage la nécessité de poursuivre les comparaisons interconfessionnelles en histoire socioreligieuse du Canada. Celles-ci permettent notamment de révéler que ce qui fut traditionnellement perçu comme l'expression de singularités n'est parfois que la manifestation de transformations sociales plus profondes vécues à une échelle plus large.

Outre une diffusion que l'on souhaiterait plus importante de ce livre au sein de la communauté scientifique nationale et internationale, on gagnerait à mettre cet ouvrage entre les mains des étudiantes et des étudiants en histoire qui sont de plus en plus sensibles aux rapports entre religion et société. Certains y puiseraient peut-être ici l'inspiration nécessaire pour entreprendre des études supérieures, au profit d'un champ de recherche qu'on souhaite fécond dans l'avenir. Il est à espérer, enfin, que le lectorat francophone soit contenté par la parution prochaine d'un ouvrage tout aussi important dans la langue de Molière. 